\title{
Prevalence and Correlates of burnout among Lebanese Health Care Workers during the COVID-19 pandemic: A national cross-sectional survey
}

Dalal Youssef ( $\nabla$ dalalyoussef.esu@gmail.com )

Ministry of Public Health: Ministere de la Sante Publique https://orcid.org/0000-0003-3085-6849

Edmond Abboud

Ministry of Public Health: Ministere de la Sante Publique

Linda Abou-Abbas

Lebanese University: Universite Libanaise

Hamad Hassan

Ministry of Public Health: Ministere de la Sante Publique

Janet Youssef

Al Zahraa University Hospital

\section{Research Article}

Keywords: Burnout, Prevalence, Correlates, COVID-19 pandemic, Health Care Workers, cross-sectional

Posted Date: January 25th, 2022

DOI: https://doi.org/10.21203/rs.3.rs-1295835/v1

License: (9) This work is licensed under a Creative Commons Attribution 4.0 International License. Read Full License 


\section{Abstract}

Background Since the COVID-19 pandemic has harshly burdened the healthcare systems, health care workers (HCWs) are at high risk of infection and confronted several stressors leading them to experience burnout. This study aimed to assess the prevalence of burnout among Lebanese health HCWs and to identify its associated factors. Methods A cross-sectional online survey was conducted between the first of November and the end of December 2020 among Lebanese HCWs working in all active hospitals distributed across the country. Using a snowball sampling technique, data was collected using an Arabic, anonymous, self-reported questionnaire comprising three sections: socio-demographic characteristics, COVID-19 exposure variables, the Copenhagen Burnout Inventory (CBI), and the threat perception scale. CBI subscale cut-off score of 50 was used to assess the prevalence of burnout among HCWs. Multinomial logistic regression analyses were performed to examine the factors associated with the different aspects of burnout. Results Out of the 1751 respondents, personal burnout (PB) was detected in its moderate and high-level aspects among $86.3 \%$ of Lebanese HCWs. Work-related burnout (WB), and client-related burnout (CB) in their moderate and high levels hit $79.2 \%$ and $83.3 \%$ of HCWs respectively. HCWs who were females, married, physicians, those who have a poor health status, and those who had specific living conditions (dependent child, elderly at home, family member with comorbidities, and a low income) were more likely to exhibit a high level of PB compared to no/low burnout. Additionally, frontline HCWs, those infected or having a colleague infected by COVID-19, and those who had a high perception of COVID-19 threat were more prone to experience a high-level PB compared to no/low burnout as well. Working in a public hospital, extensive working hours, and insufficient sleeping hours were positively associated with high PB. However, older age and altruism were negatively associated with high PB compared to no/low PB. Similar factors were found either positively or negatively associated with a high level of WB excepting health status and living conditions factors (dependent child or family member). As for $\mathrm{CB}$, older age of $\mathrm{HCW}$ ( $>30$ years) and altruism were negatively associated with high CB compared to no/low burnout. However, working in the frontline, high threat perception, extensive working hours, insufficient sleeping hours, and low income were positively associated with high CB compared to no/low burnout. Conclusion The prevalence of burnout among Lebanese HCWs was high and alarming. Enacting and implementing preventive policies and effective interventions are highly required to cultivate wellness among HCWs.

\section{Background}

The ongoing, devastating, and massive global spread of Coronavirus Disease 2019 (COVID-19) has disrupted seriously all aspects of the daily life of the general population across the globe (1-3). Furthermore, it has lifted concerns about its impact on people's mental health. Alike previous pandemics, several studies conducted in the era of COVID-19 recognized the surge in new cases of depression and anxiety and an exacerbation of existing mental health issues among exposed populations, particularly among healthcare workers (4). A multi-country study, assessing the mental health impacts of COVID-19 on healthcare workers in the Eastern Mediterranean Region (EMR) showed that $57.5 \%$ of them had 
depression, $42.0 \%$ had stress, and $59.1 \%$ had anxiety (5). However, despite the documentation of such detrimental impact of the pandemic on mental health, there is a lack of proactive actions in terms of psychological care assistance for those suffering from this crisis $(6,7)$.

Burnout emerged as one of the main mental health issues fueled by the pandemic (8). This syndrome was defined as emotional and physical exhaustion characterized by energy depletion. It resulted from prolonged and chronic exposure to stressors at the workplace which was not successfully managed (9). Although a wide range of professions and ages are affected by burnout, demanding jobs such as healthcare professions appeared to have the lion's share of this syndrome (10). In the light of the current pandemic, a worldwide increasing demand on healthcare systems was registered. Therefore, HCWs experienced, due to the nature of their work and their role in caring for COVID-19 patients, several stressors (11). These challenges included harmful working conditions, emotionally demanding patient contacts, witnessing COVID-19 related deaths, deep despondency, time pressure, extensive work hours, and work overload. All these factors are fueling HCWs' burnout at an exponential rate (12). The latter was more penetrating among those working in the frontlines of combating COVID-19 in hospitals (13). Such an increased level of burnout constitutes an occupational threat to maintaining a functioning healthcare workforce. In addition, several studies targeting burnout among HCWs have reported adverse consequences of this burnout such as the increase of the individual's risk of developing psychiatric and physical illnesses. The subsequent psychological symptoms included depression, alcohol, and drug misuse, insomnia, appetite disturbances, suicidal ideas [9-11], while the physical symptoms encompassed neck and back pain (14). However, burnout is not only threatening the wellbeing of HCWs, but may also affect the quality of health care delivered and patient safety, professional efficacy, and work performance. It also triggers HCWs' resignation, reluctance to treat people, and early retirement which may impede our recovery from the pandemic and can jeopardize the future global supply of the healthcare workforce (15). In addition, such a syndrome can even increase the risk of medical errors (16). A literature review on burnout and medical errors conducted by Brown et al., reported that many physicians suffering from burnout experienced depression and substance dependency. It is worth mentioning that HCWs' burnout may reach a tipping point with the emergence of new contagious, virulent mutants of the virus, the escalating death toll, low vaccination coverage, and slow vaccine rollout. Moreover, elevated burnout and other indicators of stress are anticipated to persist long after the pandemic.

As for health systems, low-to-middle-income countries such as Lebanon may have fewer buffering resources and capacity against shocks from the COVID-19 pandemic (17). In addition, Lebanon, this small Middle-Eastern country, is crippled by several overlapping and intractable crises with unique magnitudes on the stability of its human capital(18). Prior to the COVID-19 pandemic, a humanitarian crisis revealed by the influx of more than one million Syrian refugees to Lebanon has affected various sectors, mainly the economy, health, and education, and placed a tremendous burden on the country's health care system and health care providers. Then, the abrupt stop in capitals inflows precipitated a severe economic crisis which mirrors its sternness in systemic failures across different sectors including the healthcare system. This was the worst economic crisis in the country's history which was ranked by 
the World Bank in the top ten, possibly top three, most severe crises episodes globally since the midnineteenth century" $(19,20)$. Concurrently, the country crumbles amidst an escalated political crisis and the COVID-19 pandemic that continues to impact many aspects of life with 181503 cases and 1455 Deaths as of December 31, 2020 (21).

In such a typical context, the mental health of the general Lebanese population, particularly the one of HCWs is likely to be severely affected. One of the most significant aspects that should be considered in these conditions by occupational health professionals is burnout. Several studies conducted in Lebanon have examined burnout syndrome among residents and nurses before the pandemic (22-24). However, in the context of COVID-19, the psychological and mental health impacts of COVID-19 in Lebanon have predominantly been evaluated within specialties and single institutions (25). Furthermore, no previous study in Lebanon has focused on assessing burnout among HCWs using national, large, diverse, and multi-institution samples. Therefore, it is of great interest to assess the prevalence of burnout among Lebanese HCWs at the national level during the COVID-19 pandemic. Such information are necessary to qualify future interventions that assist stakeholders to think about strategies to reduce stressors and take care of these HCWs, as well as to direct preventive measures or prophylaxis of these morbidities during and after the pandemic.

The present study aimed to assess the prevalence of burnout among Lebanese HCWs in the context of COVID-19 and to identify its associated factors.

\section{Methods}

\section{Study design and population:}

This quantitative cross-sectional study recruited Lebanese HCWs practicing in all active health facilities located in the eight Lebanese governorates (Bekaa, Baalbeck-Hermel, South, Nabatyeh, Akkar, North, Beirut, and Mount Lebanon) using a snowball sampling technique. It was conducted over a period of two months extending from the first of November to the end of December 2020. Potential respondents were electronically invited to participate since the Lebanese government recommended minimalizing face-toface interaction. All HCWs currently working in active hospitals and who had access to the internet were eligible to participate in this study. The term "HCW" was defined as any regulated health professional and any staff member, or other essential caregivers currently working in a health care facility. Therefore, we included doctors, nurses, paramedics, and administrative staff. This study excluded HCWs who are not practicing, those who were out of the country at the time of the survey, retired HCWs, those who suffered from and those who refused to participate.

\section{Sample size calculation:}

To calculate the required sample size for this study, the Raosoft digital sample size calculator was used. Supposing that there are around 50000 registered HCWs and 40000 of them are actively practicing at the 
health facilities level, and considering a $95 \%$ confidence level and an estimation of absolute error of $5 \%$, all previous information yielded to estimate a least required sample size of 381 participants. The required sample size was achieved at an early stage before the closure of response acceptance (January 1st, 2021). Of note, we achieved a large sample size (1,751 participants) which was 4.59 times higher than the required one. Therefore, this could reduce sampling error and increase the study power.

\section{Ethical consideration:}

Participants were aware of the purpose of the study. Their participation was entirely voluntary and they were free to withdraw at any time. Since the study has no foreseeable risks and the study design assured adequate protection of the participants, written consent was obtained in an electronic format before enrolling. In addition, all information were gathered anonymously and handled confidentially. None of the survey questions asked for information that could harm the participant in any way and no reward was received by respondents in return for the participation. All methods were performed following the relevant guidelines and regulations after being reviewed and approved by the Ministry of Public Health (MOPH).

\section{Instrumentation}

Data was collected using an Arabic, online, self-administered questionnaire, developed using a google form, which included mainly closed-ended questions. The questionnaire consisted of five sections: (a) basic sociodemographic characteristics, (b) exposure to COVID-19 covariates, (c) occupational factors, and (d) the measurements.

The first section collected basic sociodemographic data of the participants, including gender, age, marital status, job category, urbanicity, health status, and living conditions. It also included questions about the history of medical illnesses and the health status of people living with the participant. Participants were also asked about the type and the location of the health facility where they worked.

The second section covered the topic of exposure to COVID-19. HCWs were queried to answer on whether they have (a) been tested for COVID-9, (b) ever been infected with COVID-19, (c) have a family member relative or colleague ever been infected by COVID-19. In addition, the status of infection protection was assessed with two items: "adherence to infection control procedures?" and "satisfaction with the hospitals' infection control precautionary measures?". Each of these variables was answered on a yes or no basis.

The third section comprises occupational factors such as the type of health facility where the HCW practice, working hours, being frontline HCW, and if the respondent treats or cares for COVID-19 patients'. Surveyed HCW was also queried about his sleep patterns during the pandemic.

The fourth section included two scales: 


\section{1-The perceived threat and altruistic acceptance of the risk questionnaire}

This instrument, which comprises 10 items, was developed by Chong et al to assess the risk perception of COVID-19. Nine of its items assessed the risk perceived by HCWs towards the COVID-19 threat and one item evaluated the risk acceptance of caring for COVID-19 cases termed as "altruism" (26). Rating of items was specified based on a five-point Likert scale ( $1=$ strongly disagree, $2=$ disagree, $3=$ neutral, $4=$ agree, $5=$ strongly angry). Responses were dichotomized into positive responses 'agree' or 'strongly agree', while 'strongly disagree', 'disagree', and 'not sure' were considered negative. This scale showed good reliability in numerous studies and was previously used in the Lebanese context (27). The Cronbach alpha of this scale in the current study was 0.721 .

\section{2- The Arabic version of Copenhagen Burnout scale A-CBI:}

The Arabic version of $\mathrm{CBI}$, validated by Youssef et al. among HCWs (28), which consisted of 19 items, was used. It evaluates personal-related (6 items), work-related (7 items), and client-related (6 items) burnout. Ratings were given based on a five-point Likert scale. Each item was scored from 0-100 ( $0=$ never, $25=$ Seldom, 0 = Sometimes, $75=$ Often, 100= Always). Of note, some questions were answered using another five-point Likert scale (to a very high degree, to a high degree, somewhat, to a low degree, to a very low degree). However, the same scoring as for the first scale was adopted. Mean items score was calculated per scale. Each scale score depicts the direction indicated by its name. To avoid the stereotypy in HCWs' responses, questions of $\mathrm{CBI}$ are mixed with other topics. To evaluate the prevalence of burnout among HCWs, a cut-off of 50 was used. A burnout level less than 50 indicated a low burnout level or its absence while a score higher than 50 indicated a moderate (50-75) and high (75-100) burnout level accordingly. In our study, the Cronbach's alpha of this scale was equal to 0.879 . Of note, a reverse coding was performed to item number 7 "Do you have enough energy for family and friends during leisure time" in the work-related burnout score.

\section{Data collection:}

After approval of the research by the Ministry of Public Health, focal persons working in health facilities were contacted via phone call and notified about the survey and its purpose. Upon their agreement to participate, an online questionnaire using a Google form was sent to them. They were also requested to disseminate the link of the study among their colleagues in the health care facility. An introductory note along with the questionnaire, which explained the intent of the survey, and an assurance that strict anonymity and confidentiality of data will be maintained. It also contains specific instructions for filling out the questionnaire. Though this has an optional field for the e-mail address of the respondents, we did not choose this option. Thus, the identity of the respondents was not known to any of the investigators. It took around 9 minutes to fill out this survey. Request to participate was sent twice at an interval of 10 days. 


\section{Statistical analysis}

The collected data was entered and analyzed using the statistical software SPSS (Statistical Package for Social Sciences), version 24.0. Descriptive statistics were reported using frequency with percentages for categorical variables and mean and standard deviation for continuous variables. Since missing data constituted $<10 \%$ of the total database, then it was not substituted. Before analysis, the distribution of each item of $\mathrm{CBI}$ and threat perception scale was checked for normality. Mean scores (mean $\pm \mathrm{SD}$ ) in personal, work-related, and client-related (pandemic related) domains were calculated using the 0 - to 100point scale. To assess the prevalence of burnout among Lebanese HCWs, CBI cut-off of 50 was used and $\mathrm{CBI}$ scores were categorized as follows: scores less than 50 are considered "no/low", scores of 50 to 74 are considered 'moderate', 75-99 are high, and a score of 100 is considered severe burnout. A bivariate analysis was conducted using the Chi2 test to test the association between $\mathrm{CBI}$ and categorical variables. Spearmen correlation was used for linear correlation between continuous variables. All variables that showed a p-value $<0.2$ in the bivariate analysis were included in the model as independent variables. Multinomial logistic analyses were performed. Statistical significance level was set at $p$-value $<0.05$

\section{Results}

\section{Socio-demographic characteristics of the surveyed HCWs:}

A total of $1751 \mathrm{HCWs}$ participated in this study. Table 1 displayed the baseline characteristics of the surveyed HCWs. The majority were female (67.3\%), married (62.6\%), and aged between 30 and 49 years old (49.4\%). More than half of the participants were living in urban areas $(61.1 \%)$ and working in private health facilities (64.9\%), located mainly in Mount-Lebanon governorate (30.8\%). The bulk of surveyed HCWs (78.5\%) had a good health status and nearly half of them had dependent children or are living at home with an elderly or a family member suffering from comorbidities (Table 1). 
Table 1: Baseline information of the surveyed Lebanese Health Care Workers $(\mathrm{N}=1751)$

n $\quad \%$

\section{Gender}

Male

572

$32.70 \%$

Female

1179

$67.30 \%$

Age (years)

$18-29$ y

636

$36.30 \%$

$30-49$ y

874

$49.40 \%$

$\geq 50 \mathrm{y}$

221

$13.70 \%$

\section{Marital status}

Single

606

$34.60 \%$

Married/Engaged

1096

$62.60 \%$

Other (Divorced or Widowed)

49

$2.80 \%$

Residence(urbanicity)

Rural

681

$38.90 \%$

Urban

1070

$61.10 \%$

\section{Occupation}

Physician

320

$18.30 \%$

Nurse

908

$51.90 \%$

Other*

523

$29.8 \%$

Health facility type

Public

615

$35.10 \%$

Private

1136

$64.90 \%$

Location of health facility

North \& Akkar

241

$13.80 \%$

Mount Lebanon

540

$30.80 \%$

Beirut

313

$17.90 \%$

South \& Nabatyeh

285

$16.30 \%$

Bekaa \& Baalbeck-Hermel

372

$21.20 \%$ 
Health status

Fair and Below

Good and above

1370

$78.20 \%$

Presence of child at home

No

779

$44.50 \%$

Yes

972

$55.50 \%$

Presence of elderly people at home

No

923

$52.70 \%$

Yes

828

$47.30 \%$

Living with a family member with comorbidities

No

782

$44.70 \%$

Yes

969

$55.30 \%$

Working in the frontline

No

735

$42.00 \%$

Yes

1016

$58.00 \%$

Following up or caring of COVID-19 case

No

843

$48.10 \%$

Yes

908

$51.90 \%$

Ever tested for COVID-19

No

389

$22.20 \%$

Yes

1362

$77.80 \%$

Personal history of COVID-19 diagnosis

No

1385

$79.10 \%$

Yes

366

$20.90 \%$

Family member ever diagnosed with COVID-19

No

1059

$60.50 \%$

Yes

692

$39.50 \%$

Colleague/friend ever diagnosed with COVID-19

No

$7.70 \%$ 
Note: n: Frequency, \% Percentage, *Other included all other health professions: pharmacists, midwives, laboratory technicians...

\section{Descriptive of the scales used in the study:}

\section{CBI items}

As seen in table 2, the items related to work burnout had the highest means such as feeling tired from every working hour $(68.11 \pm 15.53)$, being frustrated from work $(67.96 \pm 16.43)$, and feeling worn out at the end of the working day $(67.804 \pm 16.636)$. The items related to personal burnout came in the second rank, such as being emotionally or physically exhausted, feeling worn out, feeling tired or susceptible to illness, and thinking about the lack of ability to take anymore. In regards to internal consistency, the used scales showed good reliability and the a-values obtained of subscales ranged between 0.721 and 0.903 , indicating good reliability: CBI ( $a=0.861)$, PB ( $a=0.814)$; WB ( $a=0.903) ; C B(a=0.834)$ and TP $(a=0.721)$. The overall $\mathrm{CBI}$ had a mean of 63.65 (SD=21.32) while the value for the TP scale was $35.38(S D=2.66)$. The highest burnout mean was shown in WB aspect $(67.53 \pm 17.15)$ followed by PB $(65.09 \pm 17.33)$ and CB (63.65 \pm 21.32$)$. The normality of all used scales and subscales was assumed since skewness and kurtosis were lower than 1 and the sample size was larger than 300 . 
Table 2: Descriptive statistics of $\mathrm{CBI}$ and threat perception

\begin{tabular}{|c|c|c|}
\hline & Mean & $\begin{array}{l}\text { Std. } \\
\text { Deviation }\end{array}$ \\
\hline Personal burnout (nb of items $=6, a=0.814$ ) & 65.09 & 17.23 \\
\hline How often do you feel weak and susceptible to illness? & 65.43 & 17.21 \\
\hline How often you are emotionally exhausted? & 64.52 & 17.09 \\
\hline How often do you feel worn out? & 65.67 & 17.52 \\
\hline How often do you feel tired? & 63.89 & 17.15 \\
\hline How often you are physically exhausted? & 64.13 & 17.03 \\
\hline How often do you think: "I can't take it anymore"? & 66.93 & 14.57 \\
\hline Work burnout (nb of items $=7, a=0.903$ ) & 67.53 & 17.15 \\
\hline Is your work emotionally exhausting? & 67.43 & 16.45 \\
\hline Does your work frustrate you? & 67.96 & 16.43 \\
\hline Do you feel worn out at the end of the working day? & 67.81 & 16.64 \\
\hline Do you feel that every working hour is tiring for you? & 68.11 & 15.53 \\
\hline Are you exhausted in the morning at the thought of another day at work? & 67.54 & 16.56 \\
\hline Do you feel burnt out because of your work? & 67.11 & 15.33 \\
\hline Do you have enough energy for family and friends during leisure time? & 66.76 & 16.11 \\
\hline Client Burnout ( $n b$ of items $=6, a=0.834$ ) & 57.70 & 22.10 \\
\hline Do you find it frustrating to work with clients? & 56.05 & 21.71 \\
\hline Does it drain your energy to work with clients? & 56.44 & 21.49 \\
\hline Do you find it hard to work with clients? & 55.25 & 22.39 \\
\hline $\begin{array}{l}\text { Do you sometimes wonder how long you will be able to continue working } \\
\text { with clients? }\end{array}$ & 52.69 & 21.10 \\
\hline $\begin{array}{l}\text { Do you feel that you give more than you get back when you work with } \\
\text { clients? }\end{array}$ & 55.57 & 22.13 \\
\hline Are you tired of working with clients? & 70.09 & 21.01 \\
\hline Threat perception ( $n b$ of items $=10,, a=0.721$ ) & 35.38 & 2.66 \\
\hline
\end{tabular}




\section{Threat perception and altruistic acceptance of risk during the outbreak}

Nearly $90 \%$ of surveyed HCWs believed that their job was putting them at risk, felt extra stress at work, and were afraid to transmit the COVID-19 to others. In addition, $81.6 \%$ were afraid of being infected by COVID-19 and $77.3 \%$ of them felt that they had little control over being infected or not. Only $7.9 \%$ of participants perceived a little chance of survival if they got infected and $3 \%$ thought about resigning because of COVID-19. Also, $88.6 \%$ of surveyed HCWs considered that their families and friends feared getting ill because of them. More than half of respondents were concerned about the avoidance of their families by others due to the nature of their work. As for altruistic acceptance of risks, the majority of participants (77.7\%) accepted taking the risk of caring for COVID-19 patients (Figure 1).

\section{Prevalence of burnout among Lebanese HCWs}

Out of all, personal burnout was detected in its moderate and high-level aspects among $86.3 \%$ of Lebanese HCWs with more than half (52.5\%) of them exhibiting a high level of personal burnout (CBI score $>75$ ). In regards to WB, a moderate and high level of burnout hits around $79.2 \%$.of HCWs. Notably, $47 \%$ of them suffered from a high level of WB. Moderate and High levels of CB were prevalent among $83.3 \%$ of HCWs and $35.4 \%$ of them experienced a high level of CB. Of note, only a small percentage ( $\leq$ $20 \%$ ) of surveyed HCWs, experienced a low level of burnout or didn't s burnout at all.

\section{Factors associated with Personal burnout among HCWs:}

\section{1-Moderate PB versus no/low PB:}

Being married compared to single/divorced $(\mathrm{aOR}=1.60 ; 95 \% \mathrm{Cl} 1.21-1.90)$, being a physician compared to other HCWs' occupations $(\mathrm{aOR}=2.59 ; 95 \% \mathrm{Cl} 2.01-6.72)$, having a dependent child $(\mathrm{aOR}=1.15 ; 95 \% \mathrm{Cl}$ $1.61-2.19)$, working in the frontlines $(\mathrm{aOR}=1.82 ; 95 \% \mathrm{Cl} 1.62-5.12)$, being diagnosed with COVID -19 $(\mathrm{aOR}=1.92 ; 95 \% \mathrm{Cl} 1.28-3.48)$ or having a colleague diagnosed with COVID-19 $(\mathrm{aOR}=2.24 ; 95 \% \mathrm{Cl}$ 1.51-6.24), higher COVID-19 threat perception ( $\mathrm{aOR}=1.52 ; 95 \% \mathrm{Cl} 1.22-3.27)$, and limited sleeping hours $(\mathrm{aOR}=1.85 ; 95 \% \mathrm{Cl} 1.12-3.92)$ were significantly positively associated with moderate personal burnout compared to no/low PB (Table 3, Model 1).

\section{2- High PB versus no/low PB:}

As for socio-demographic factors, being female (aOR $=1.78 ; 95 \% \mathrm{Cl} 1.25-2.77)$, married compared to single/divorced $(\mathrm{aOR}=1.51 ; 95 \% \mathrm{Cl} 1.22-3.18)$, having a poor health status $(\mathrm{aOR}=1.80 ; 95 \% \mathrm{Cl} 1.11-$ 3.55) were significantly positively associated with high personal burnout compared to no/low burnout. In addition, having a dependent child $(\mathrm{aOR}=1.51 ; 95 \% \mathrm{Cl} 1.13-4.89)$ or an elderly at home $(\mathrm{aOR}=2.09$; $95 \% \mathrm{Cl} 1.28-5.56)$, having a family member suffering from comorbidities(aOR $=1.62 ; 95 \% \mathrm{Cl} 1.24-5.53$ ), and a low income compared to high/moderate outcome (aOR $=3.43 ; 95 \% \mathrm{Cl} 1.38-4.62)$ ), were significantly positively associated with high personal burnout compared to no/low burnout. However, 
older age ( $>30$ years) compared to younger age ( $\leq 30$ years) $(\mathrm{aOR}=0.81 ; 95 \% \mathrm{Cl} 0.54-0.93)$ was negatively associated with high PB compared to no/low PB.

As for exposure factors, working in the frontlines ( $\mathrm{aOR}=1.36 ; 95 \% \mathrm{Cl} 1.12-4.01)$, being diagnosed with COVID-19 $(\mathrm{aOR}=2.17 ; 95 \% \mathrm{Cl} 1.82-3.66)$ or having a colleague diagnosed with COVID-19 $(\mathrm{aOR}=1.66$; $95 \% \mathrm{Cl} 1.47-5.93)$ and higher COVID-19 threat perception (aOR $=1.42 ; 95 \% \mathrm{Cl} 1.20-3.19)$ as well, increased the likelihood of high PB compared to no/low burnout. In terms of work-related factors, being a physician compared to other HCWs' occupations ( $\mathrm{aOR}=2.83 ; 95 \% \mathrm{Cl} 1.12-6.98)$, working in a public health facility $(\mathrm{aOR}=3.29 ; 95 \% \mathrm{Cl} 2.84-5.32)$, extensive working hours $(\mathrm{aOR}=2.03 ; 95 \% \mathrm{Cl} 1.48-4.01)$, and limited sleeping hours $(\mathrm{aOR}=1.85 ; 95 \% \mathrm{Cl} 1.12-3.92)$, were significantly positively associated with high personal burnout compared to no/low burnout. However, altruism (aOR $=0.46 ; 95 \% \mathrm{Cl} 0.33-$ 0.71)was associated negatively with high PB (Table 3, Model 2). 
Table 3: Multivariable analysis: multinomial regression for the personal burnout among HCWs 95\% Confidence interval

Variable P- aOR Lower Bound Upper Bound value

Model 1: Personal burnout (Moderate versus low/no burnout)

\begin{tabular}{|c|c|c|c|c|}
\hline Age $\left(>30\right.$ y vs $\left.<30 Y^{\star}\right)$ & 0.220 & 1.820 & 0.715 & 2.767 \\
\hline Marital status (Married vs single/divorced*) & 0.049 & 1.604 & 1.212 & 1.902 \\
\hline Occupation (physicians vs other HCWs *) & 0.022 & 2.590 & 2.013 & 6.724 \\
\hline Hospital type (Private vs Public*) & 0.257 & 0.612 & 0.558 & 1.917 \\
\hline Health insurance (Private vs Public*) & 0.336 & .719 & 0.841 & 5.933 \\
\hline Gender (Female vs male*) & 0.213 & 1.018 & 0.886 & 1.386 \\
\hline Health condition (Poor vs Good*) & 0.207 & 1.773 & 0.946 & 2.290 \\
\hline Presence of child at home (yes vs no*) & 0.026 & 1.154 & 1.606 & 2.194 \\
\hline Presence of elderly at home (yes vs no*) & 0.181 & 0.987 & 0.831 & 1.978 \\
\hline Family member with comorbidities & 0.231 & 0.866 & 0.710 & 2.012 \\
\hline Working in the frontline (yes vs no*) & 0.025 & 1.822 & 1.615 & 5.117 \\
\hline Tested for COVID19 (PCR test) (yes vs no*) & 0.601 & 0.957 & 0.713 & 6.816 \\
\hline Diagnosed as COVID19 case (yes vs no*) & 0.016 & 1.915 & 1.277 & 3.481 \\
\hline Family member with comorbidities (yes vs no*) & 0.183 & 0.897 & 0.211 & 3.822 \\
\hline Colleague diagnosed with COVID19 (yes vs no*) & $<0.001$ & 2.238 & 1.509 & 6.239 \\
\hline Threat perception scale & 0.038 & 1.517 & 1.218 & 3.271 \\
\hline Altruistic (yes vs no *) & 0.149 & 0.435 & 0.377 & 1.615 \\
\hline Extensive working hours (yes vs no*) & 0.198 & 0.886 & 0.765 & 1.245 \\
\hline Income (Low vs high*) & 0.031 & 0.698 & 0.437 & 0.721 \\
\hline Sleeping hours (less than 6 hours vs $>6$ hours *) & 0.011 & 1.854 & 1.123 & 3.922 \\
\hline
\end{tabular}

Model 2: Personal burnout (High versus low/no burnout)

\begin{tabular}{lllll} 
Age $\left(<30\right.$ Y vs $>30$ y vs ${ }^{*}$ ) & 0.011 & 1.812 & 1.541 & 2.929 \\
\hline Marital status (Married vs single/divorced*) & 0.041 & 1.513 & 1.218 & 3.183 \\
\hline Occupation (physicians vs other HCWs *) & 0.003 & 2.832 & 1.122 & 6.983 \\
\hline Hospital type (Public vs Private *) & 0.038 & 3.291 & 2.837 & 5.316
\end{tabular}




\begin{tabular}{|c|c|c|c|c|}
\hline Health insurance (Private vs Public*) & 0.195 & 1.713 & 0.896 & 2.357 \\
\hline Gender (Female vs male*) & 0.025 & 1.776 & 1.250 & 2.075 \\
\hline Health condition (Poor vs Good*) & 0.029 & 1.804 & 1.114 & 3.549 \\
\hline Presence of child at home (yes vs no*) & 0.016 & 1.507 & 1.130 & 4.890 \\
\hline Presence of elderly at home (yes vs no*) & 0.049 & 2.091 & 1.277 & 5.556 \\
\hline Family member with comorbidities & 0.327 & 1.017 & 0.715 & 1.445 \\
\hline Working in the frontline (yes vs no*) & 0.040 & 1.365 & 1.119 & 4.013 \\
\hline Tested for COVID19 (PCR test) (yes vs no*) & 0.656 & 0.990 & 0.687 & 1.426 \\
\hline Diagnosed as COVID19 case (yes vs no*) & 0.039 & 1.833 & 1.552 & 6.260 \\
\hline Family member with comorbidities (yes vs no*) & 0.034 & 1.618 & 1.236 & 5.525 \\
\hline Colleague diagnosed with COVID19 (yes vs no*) & 0.015 & 1.664 & 1.476 & 3.925 \\
\hline Threat perception scale & 0.026 & 1.420 & 1.196 & 3.189 \\
\hline Altruistic (yes vs no*) & 0.011 & 0.457 & 0.328 & 0.712 \\
\hline Extensive working hours (yes vs no*) & $<0.001$ & 2.031 & 1.476 & 4.012 \\
\hline Income (Low vs high*) & 0.041 & 3.435 & 1.377 & 4.615 \\
\hline Sleeping hours (less than 6 hours vs $>6$ hours *) & 0.023 & 2.513 & 1.428 & 5.811 \\
\hline
\end{tabular}

* Reference group, numbers in bold indicate significant p-value, aOR: adjusted odds ratio, Goodness of fit Pearson value $=2997.382, p<0.001$, Pseudo R2=13.9

\section{Factors associated with Work-related burnout among HCWs:}

\section{1-Moderate WB versus no/low WB:}

HCWs who were physicians compared to those with other healthcare occupations $(a O R=1.95 ; 95 \% \mathrm{Cl}$ 1.34-4.41), those who worked in a public health facility compared to HCWs practicing private health facility $(\mathrm{aOR}=1.82 ; 95 \% \mathrm{Cl} 1.42-3.27)$ and those who have a colleague diagnosed with COVID-19 (aOR = $2.31 ; 95 \% \mathrm{Cl} 1.97-3.44)$ were more likely to suffer from a moderate level of WB than from a no/low PB level (Table 4, Model 1).

\section{2- High WB versus no/low WB:}

As for socio-demographic factors, being female HCW (aOR $=2.78 ; 95 \% \mathrm{Cl} 1.15-4.08)$, married $(\mathrm{aOR}=$ $2.20 ; 95 \% \mathrm{Cl} 1.40-3.98)$, aged 30 years old or less ( $\mathrm{aOR}=2.78 ; 95 \% \mathrm{Cl} 1.15-4.08)$ were significantly positively associated with high WB compared to no/low burnout. In addition, low income compared to high/moderate outcome $(\mathrm{aOR}=3.01 ; 95 \% \mathrm{Cl} 1.52-7.31)$ ), were significantly positively associated with 
high personal burnout compared to no/low burnout. However, older age (>30 years) compared to younger age $(\leq 30$ years $)(\mathrm{aOR}=0.81 ; 95 \% \mathrm{Cl} 0.54-0.93)$ was negatively associated with high $\mathrm{PB}$ compared to no/low PB.

As for exposure factors, working in the frontlines $(\mathrm{aOR}=2.17 ; 95 \% \mathrm{Cl} 1.82-5.66)$, having a colleague diagnosed with COVID-19 $(\mathrm{aOR}=1.66 ; 95 \% \mathrm{Cl} 1.17-3.92)$ and higher COVID-19 threat perception (aOR = $2.42 ; 95 \% \mathrm{Cl} 1.20-5.19$ ) as well, increased the likelihood of high WB compared to no/low burnout. In terms of work-related factors, being a physician compared to other HCWs' occupations ( $\mathrm{aOR}=2.51 ; 95 \%$ $\mathrm{Cl} 1.15-3.08)$, working in a public health facility $(\mathrm{aOR}=3.19 ; 95 \% \mathrm{Cl} 2.11-6.32)$, extensive working hours $(\mathrm{aOR}=2.71 ; 95 \% \mathrm{Cl} 1.85-4.79)$, and limited sleeping hours $(\mathrm{aOR}=2.51 ; 95 \% \mathrm{Cl} 1.43-4.81)$, were significantly positively associated with high WB compared to no/low burnout. However, altruism (aOR = $0.65 ; 95 \% \mathrm{Cl} 0.41-0.61$ )was associated negatively with high WB (Table 4, Model 2). 
Table 4: Multivariable analysis: multinomial regression for the work-related burnout among HCWs

95\% Confidence interval

Variable

P-value aOR Lower Bound Upper Bound

Model 1: Work-related burnout (Moderate versus low/no burnout)

\begin{tabular}{lllll} 
Age $\left(>30\right.$ y vs $<30 Y^{\star}$ ) & 0.210 & 1.530 & 0.902 & 2.183 \\
\hline Marital status (Married vs single/divorced*) & 0.248 & 1.442 & 0.715 & 1.987 \\
\hline Occupation (physicians vs other HCWs*) & $\mathbf{0 . 0 2 2}$ & $\mathbf{1 . 9 5 0}$ & $\mathbf{1 . 3 3 8}$ & $\mathbf{4 . 4 0 5}$ \\
\hline Hospital type (Public vs Private $\left.{ }^{*}\right)$ & $\mathbf{0 . 0 1 7}$ & $\mathbf{1 . 8 2 0}$ & $\mathbf{1 . 4 1 8}$ & $\mathbf{3 . 2 7 0}$ \\
\hline Health insurance (Private vs Public*) & 0.136 & 0.826 & 0.842 & 3.922 \\
\hline Gender (Female vs male*) & 0.318 & 1.183 & 0.886 & 3.663 \\
\hline Health condition (Poor vs Good*) & 0.119 & 1.773 & 0.946 & 2.290 \\
\hline Presence of child at home (yes vs no*) & 0.126 & 1.154 & 0.606 & 2.196 \\
\hline Presence of elderly at home (yes vs no*) & 0.141 & 1.185 & 0.616 & 2.277 \\
\hline Family member with comorbidities & 0.183 & 0.960 & 0.504 & 1.828 \\
\hline Working in the frontline (yes vs no*) & 0.225 & 1.909 & 0.651 & 2.571 \\
\hline Tested for COVID19 (PCR test) (yes vs no*) & 0.668 & 0.985 & 0.534 & 1.816 \\
\hline Diagnosed as COVID19 case (yes vs no*) & 0.121 & 0.751 & 0.421 & 4.846
\end{tabular}

Family member with comorbidities (yes vs no*)

$\begin{array}{llll}0.283 & 0.897 & 0.211 & 3.822\end{array}$

Colleague diagnosed with COVID19 (yes vs no*)

$\begin{array}{llll}<.001 & 2.310 & 1.967 & 3.439\end{array}$

Threat perception scale

$\begin{array}{llll}0.208 & 1.657 & 0.941 & 4.712\end{array}$

Altruistic (yes vs no*)

$0.301 \quad 0.435$

0.377

1.615

Extensive working hours (yes vs no*)

$0.091 \quad 0.681 \quad 0.465$

3.451

Income (high vs Low *)

$0.131 \quad 0.698$

0.437

2.712

Sleeping hours ( $>6$ hours vs less than 6 hours*)

$\begin{array}{lll}0.171 & 0.654 & 0.713\end{array}$

2.917

Model 2: Work-related burnout (High versus no/low burnout)

\begin{tabular}{lllll} 
Age $\left(>30\right.$ y vs $\leq 30 Y^{*}$ ) & $\mathbf{0 . 0 1 2}$ & 0.770 & 0.608 & 0.974 \\
\hline Marital status (Married vs single/divorced*) & $\mathbf{0 . 0 0 6}$ & 2.203 & 1.401 & 3.983 \\
\hline Occupation (physicians vs other HCWs *) & $\mathbf{0 . 0 0 9}$ & 2.513 & 1.147 & 4.983 \\
\hline Hospital type (Private vs Public*) & $<0.001$ & 3.191 & 2.113 & 6.316
\end{tabular}




\begin{tabular}{lllll}
\hline Health insurance (Private vs Public*) & 0.371 & 0.885 & 0.676 & 1.157 \\
\hline Gender (Female vs male*) & $\mathbf{0 . 0 2 5}$ & 2.776 & 1.150 & 4.075 \\
\hline Health condition (Poor vs Good*) & 0.222 & 1.044 & 0.704 & 1.549 \\
\hline Presence of child at home (yes vs no*) & 0.306 & 1.507 & 0.813 & 3.890 \\
\hline Presence of elderly at home (yes vs no*) & 0.131 & 3.099 & 0.977 & 6.556 \\
\hline Family member with comorbidities & 0.927 & 1.017 & 0.715 & 1.445 \\
\hline Working in the frontline (yes vs no*) & $\mathbf{0 . 0 4 0}$ & $\mathbf{2 . 1 6 5}$ & $\mathbf{1 . 8 1 7}$ & $\mathbf{5 . 6 6 0}$ \\
\hline Tested for COVID19 (PCR test) (yes vs no*) & 0.656 & 0.990 & 0.687 & 1.426 \\
\hline Diagnosed as COVID19 case (no vs yes*) & 0.069 & 0.833 & 0.552 & 1.260 \\
\hline Family member with comorbidities (yes vs no*) & 0.324 & 1.618 & 0.836 & 3.525 \\
\hline Colleague diagnosed with COVID19 (yes vs no*) & $\mathbf{0 . 0 1 5}$ & $\mathbf{1 . 6 6 4}$ & $\mathbf{1 . 1 7 6}$ & $\mathbf{3 . 9 2 4}$ \\
\hline Threat perception scale & $\mathbf{0 . 0 2 6}$ & $\mathbf{2 . 3 1 1}$ & $\mathbf{1 . 8 1 6}$ & $\mathbf{5 . 1 8 9}$ \\
\hline Altruistic (yes vs no*) & $\mathbf{0 . 0 1 1}$ & $\mathbf{0 . 6 5 1}$ & $\mathbf{0 . 4 0 8}$ & $\mathbf{0 . 6 1 2}$ \\
\hline Extensive working hours (yes vs no*) & $<\mathbf{0 . 0 0 1}$ & $\mathbf{2 . 7 1 3}$ & $\mathbf{1 . 8 4 7}$ & $\mathbf{4 . 7 9 2}$ \\
\hline Income (Low vs high*) & $\mathbf{0 . 0 1 1}$ & $\mathbf{3 . 0 1 4}$ & $\mathbf{1 . 5 2 2}$ & $\mathbf{7 . 3 1 4}$ \\
\hline Sleeping hours (less than 6 hours vs >6 hours *) & $\mathbf{0 . 0 2 3}$ & $\mathbf{2 . 5 1 3}$ & $\mathbf{1 . 4 2 8}$ & $\mathbf{6 . 8 1 1}$ \\
\hline * Reference group, numbers in bold indicate significant p-value, a0P: adjusted odds ratio, Goodness of \\
fit Pearson value=2103.19, p<0.001, Pseudo R2=11.4
\end{tabular}

\section{Factors associated with Client-related burnout among HCWs:}

\section{1-Moderate CB versus no/low CB:}

Working in the frontlines $(\mathrm{aOR}=1.91 ; 95 \% \mathrm{Cl} 1.65-2.57)$, having a colleague diagnosed with COVID-19 $(\mathrm{aOR}=2.31 ; 95 \% \mathrm{Cl} 1.97-3.24)$, and higher COVID-19 threat perception $(\mathrm{aOR}=1.35 ; 95 \% \mathrm{Cl} 1.30-3.12)$, and limited sleeping hours $(\mathrm{aOR}=1.85 ; 95 \% \mathrm{Cl} 1.12-3.92)$ were significantly positively associated with moderate personal burnout compared to no/low CB (Table 5, Model 1).

\section{2- High CB versus no/low CB:}

Older age of $\mathrm{HCW}(>30$ years) $(\mathrm{aOR}=0.77 ; 95 \% \mathrm{Cl} 0.61-0.97)$ and altruism $(\mathrm{aOR}=0.52 ; 95 \% \mathrm{Cl} 0.41-$ 0.87) were negatively associated with high $\mathrm{CB}$ compared to no/low burnout. However, working in the frontline $(\mathrm{aOR}=2.01 ; 95 \% \mathrm{Cl} 1.50-4.13)$, threat perception $(\mathrm{aOR}=1.42 ; 95 \% \mathrm{Cl} 1.50-4.13)$, extensive working hours $(\mathrm{aOR}=2.13 ; 95 \% \mathrm{Cl} 1.49-4.00)$, less than 6 hours sleeping hours $(\mathrm{aOR}=1.57 ; 95 \% \mathrm{Cl}$ $1.21-3.11)$, and low income compared to high/moderate outcome (aOR $=1.91 ; 95 \% \mathrm{Cl} 1.42-3.27)$ ), were 
significantly positively associated with high client burnout compared to no/low burnout (Table 5, Model 2). 
Table 5: Multivariable analysis: multinomial regression for the client-related burnout among HCWs

95\% Confidence interval

Variable

$\begin{array}{llll}\mathrm{P}- & \text { aOR } & \begin{array}{l}\text { Lower } \\ \text { Bound }\end{array} & \begin{array}{l}\text { Upper } \\ \text { value }\end{array}\end{array}$

Model 1: client-related burnout (Moderate versus low/no burnout)

Age $\left(>30\right.$ y vs $\left.<30 Y^{*}\right)$

0.313

1.713

0.922

3.018

Marital status (Married vs single/divorced*)

0.418

1.314

0.507

1.807

Occupation (physicians vs other HCWs *)

$0.152 \quad 2.590$

0.839

2.705

Hospital type (Private vs Public*)

0.302

0.812

0.701

1.327

Gender (Female vs male*)

0.308

1.183

0.886

4.643

Health condition (Poor vs Good*)

0.119

1.773

0.946

3.139

Presence of child at home (yes vs no*)

0.298

1.154

0.816

2.196

Presence of elderly at home (yes vs no*)

$0.111 \quad 1.185 \quad 0.616$

2.277

Family member with comorbidities

0.181

0.960

0.504

1.828

Working in the frontline (yes vs no*)

$\begin{array}{llll}\mathbf{0 . 0 2 5} & 1.909 & 1.651 & 2.571\end{array}$

Diagnosed as COVID19 case (yes vs no*)

0.112

2.351

0.814

4.846

Family member with comorbidities (yes vs no*)

0.283

0.897

0.211

3.822

Colleague diagnosed with COVID19 (yes vs no*)

$<0.001 \quad 2.310$

1.967

3.239

Threat perception scale

$\mathbf{0 . 0 4 8} \quad 1.345 \quad 1.298$

3.124

Altruistic (yes vs no*)

0.241

0.435

0.377

1.615

Extensive working hours (yes vs no*)

$0.098 \quad 0.886$

0.765

1.245

Income (Low vs high*)

$0.126 \quad 0.898$

0.753

1.942

Sleeping hours ( $>6$ hours vs less than 6 hours*)

$\begin{array}{lll}0.271 & 0.854 & 0.723\end{array}$

1.914

\section{Model 2: client-related burnout (High versus no/low burnout)}

Age $\left(>30\right.$ y vs $\left.<30 Y^{*}\right)$

0.029

0.770

0.608

0.974

Marital status (Married vs single/divorced*)

0.152

1.513

0.772

4.003

Occupation (physicians vs other HCWs *)

$0.112 \quad 1.093 \quad 0.981$

2.832

Hospital type (Private vs Public*)

0.331

2.291

0.811

4.022

Gender (Female vs male*)

0.205

1.776

1.115

2.075

Health condition (Poor vs Good*)

$0.329 \quad 1.044 \quad 0.704$

1.905 


\begin{tabular}{lllll} 
Presence of child at home (yes vs no*) & 0.116 & 1.507 & 0.893 & 3.890 \\
\hline Presence of elderly at home (yes vs no*) & 0.449 & 2.099 & 1.277 & 2.556 \\
\hline Family member with comorbidities & 0.527 & 1.017 & 0.895 & 10.215 \\
\hline Working in the frontline (yes vs no*) & $\mathbf{0 . 0 2 1}$ & $\mathbf{2 . 0 1 3}$ & $\mathbf{1 . 5 0 2}$ & $\mathbf{4 . 1 3 1}$ \\
\hline Diagnosed as COVID19 case (yes vs no*) & 0.308 & 1.833 & 0.552 & 3.260 \\
\hline Family member with comorbidities (yes vs no*) & 0.141 & 1.618 & 0.936 & 2.145 \\
\hline Colleague diagnosed with COVID19 (yes vs no*) & 0.315 & 0.664 & 0.541 & 2.912 \\
\hline Threat perception scale & $\mathbf{0 . 0 3 1}$ & $\mathbf{1 . 4 2 0}$ & $\mathbf{1 . 1 9 6}$ & $\mathbf{2 . 1 8 9}$ \\
\hline Altruistic (yes vs no*) & $<0.001$ & $\mathbf{0 . 5 1 7}$ & $\mathbf{0 . 4 1 2}$ & $\mathbf{0 . 8 6 6}$ \\
\hline Extensive working hours (yes vs no*) & $<0.001$ & $\mathbf{2 . 1 3 1}$ & $\mathbf{1 . 4 7 6}$ & $\mathbf{4 . 0 0 2}$ \\
\hline Income (Low vs high*) & $\mathbf{0 . 0 0 6}$ & $\mathbf{1 . 9 0 5}$ & $\mathbf{1 . 4 1 5}$ & $\mathbf{3 . 2 7 1}$ \\
\hline Sleeping hours (less than 6 hours vs >6 hours vs & $\mathbf{0 . 0 2 3}$ & $\mathbf{1 . 5 7 3}$ & $\mathbf{1 . 2 0 9}$ & $\mathbf{3 . 1 0 8}$ \\
\hline *) & & & & \\
\hline
\end{tabular}

* Reference group, numbers in bold indicate significant p-value, aOP: adjusted odds ratio

\section{Discussion}

To fulfill their dedication to the medical profession's responsibilities and obligations, HCWs have done their utmost around the clock to save, diagnose, and treat COVID-19 patients. The outbreak of COVID-19 triggered widespread alarm about the potential of this unprecedented global crisis to increase the level of burnout among HCWs. Therefore, this study is the first national, large, diverse, and multi-institution study assessing the prevalence of burnout among HCWs and identifying its associated factors during the COVID-19 pandemic, using a validated scale. We believe that this study outlines interesting avenues for future research.

Our study found that around $80 \%$ of HCWs suffered from a moderate and high level of burnout comprising its three dimensions (Personal, occupational and patient-related). HCWs who were females, married, physicians, those who have a poor health status, and those who had specific living conditions (dependent child, elderly at home, family member with comorbidities, and a low income) were more likely to exhibit a high level of PB compared to no/low burnout. Additionally, frontline HCWs, those who were diagnosed with COVID-19 or had a COVID-19, and those who had a high perception of COVID-19 threat were more prone to suffer a high-level PB compared to no/low burnout as well. Working in a public health facility, extensive working hours, and limited sleeping hours were significantly positively associated with high PB. However, older age and altruism were negatively associated with high PB compared to no/low PB. The same factors were found either positively or negatively associated with a high level of WB compared to no/low WB except health status and living conditions (dependent child or family member). 
As for high CB level, Older age of HCW (>30 years) and altruism were negatively associated with high CB compared to no/low burnout. However, working in the frontline, threat perception, extensive working hours, sleeping hours (less than 6 hours), and low income were significantly positively associated with high CB compared to no/low burnout. We found a high prevalence of burnout during the current pandemic compared to the pre-COVID-19 era. This is difficult to compare with previous literature as most studies had used different scales.

The burnout level found among Lebanese HCWs was alarming since more than third-quarter of them suffered from all aspects of burnout including personal (86.3\%), work-related (79.2\%), and client-related burnout (83.3\%) in their moderate and high levels. The present study lacked a control group but our results supported the findings of various studies regarding the potential of the pandemic to increase the level of burnout among HCWs. Similarly, a study conducted in China showed also that HCWs battling COVID-19 exhibited a high level of burnout (29). However, the worrying prevalence of burnout found in our study was higher than the figures reported in previous studies conducted among HCWs in Asia during the COVID-19 pandemic as these studies disclosed that the prevalence of burnout in HCWs varies from $31.4 \%$ to $75 \%$ (29-32). On the other hand, our results were in line with the findings of a study conducted among HCWs in Saudi Arabia, an Arabic country that owns one of the superlative healthcare systems in the Middle East (33), where 75\% of Saudi HCWs suffered from burnout during the COVID-19 pandemic (32). In comparison with Europe which was severely impacted by COVID-19, several studies conducted by Barello et al.(34) and Lasalvia et al.(35) showed that Italian HCWs revealed significant work-related burnout symptoms and $56 \%$ of them reported emotional exhaustion (36). In Portugal, According Duarte I et al. concluded that more than half of HCWs had symptoms of personal burnout (37). Of note, owing to both the COVID-19 pandemic and severe economic crisis, such a high prevalence of burnout among Lebanese HCWs was anticipated. A similar finding was reported in the Libyan context, where $67.1 \%$ of HCWs suffered from emotional exhaustion (38) due to the overlapping crises revealed by the pandemic and the civil war). It is noteworthy that the large difference across these studies in terms of the prevalence of burnout was expected since it could be resulting from the assessment methods' heterogeneity, the disparity in burnout definitions, and regional differences (39).

A peculiar finding in our study was the drastic change in the dynamic of burnout during the pandemic. A huge increase in the prevalence of client-related burnout (pandemic-related) increased (83.3\%) among HCWs was recorded in the era of COVID-19 compared to previous studies. For example, a pre-COVID19 study conducted by Žutautienè et al. found a low prevalence of CB $(35.1 \%)$ compared to the PB $(44.8 \%)$ and WB (46.7\%) burnout (40). For the personal aspect of burnout, it was predominant. Of note, such a high prevalence of PB should not be linked completely to COVID-19 related factors given that other veiled risk factors could be associated with the increase of PB such as the economic factors.

In terms of factors associated with high PB, HCWs who were females, married, physicians, those who have a poor health status, and those who had specific living conditions (dependent child, elderly at home, family member with comorbidities, and a low income) were more likely to exhibit a high level of PB compared to no/low burnout. As for gender, while some studies agree on the fact that there is no real 
effect of this variable in the occurrence of burnout $(41,42)$, the data from the Medscape National Physician Report indicate that women physicians reported more often symptoms of burnout (40). Our findings were also consistent with the results of an Italian survey that found higher levels of burnout in females and in young (aged $<30$ years) HCWs (43). Furthermore, a recent systematic review conducted by Prasad (44) showed higher stress scores in US health organizations among women. This could be explained by the high exposure to risk for female HCWs given their predominance in patient-facing roles, gender expectations in care, with high workloads at their home. As for occupation, despite that Burnout has been shown to occur in all kinds of jobs, our study showed that physicians were more likely to experience a high level of personal and occupational burnout than other HCWs. A similar finding was reported by Shanafelt et al who reported that the incidence of burnout was $37.9 \%$ in physicians compared to $27.8 \%$ in the control population $(p<0.001)(45)$.

Another important aspect of personal and occupational burnout found in our study was the "married" marital status of HCWs. In addition, living conditions such as having a dependent child, elderly or family member suffering from comorbidities at home were associated with a higher level of PB. The high prevalence of burnout could be resulting from family demands that denote possible familial-related responsibilities such as caring for family members who are sick, childcare, providing support, and managing complex familial relationships $(46,47)$. Of note, family demands are commonly gendered as a result of imposed roles on women $(48,49)$ and there are discrepancies in who bears the brunt of such demands. . Studies show that HCWs are tormented when it comes to balancing providing care for their patients and their families, which can lead to impairments in both spheres (50) as well as to the burnout of HCWs.

Our study found that HCWs who worked in the frontline and those who were directly involved in the diagnosis and treatment of COVID-19 cases were more likely to express a high level of personal and occupational burnout. This was also reported in a study conducted in China comparing the mental health disturbances in physicians and nurses working at the frontline and the second-line healthcare workers (51). In addition to burnout, other studies disclosed also a higher incidence and more severe symptoms of depression, anxiety, insomnia, and mental distress among frontline HCWs. Additionally, HCWs who had a high perception of COVID-19 threat were more prone to suffer from a high-level PB compared to no/low burnout as well. In this context, several studies focusing on conventional risks supported the potential association between perceived risk and negative outcomes for the individual, such as job burnout and low job satisfaction (52-54). Overall, based on the aforementioned results of previous research, we assumed that the perceived risk of being infected by COVID-19 at work and the fear of being infected were positively associated with a high level of burnout in its different aspects (personal, work-related, or patient-related). As for the history of COVID-19 infection and having a colleague infected with COVID-19, one multicenter cross-sectional survey also has shown that the history of contact with the patient was a risk factor doubling the risk of negative mental health outcomes such as anxiety and depression during the COVID-19 pandemic (55). 
Another peculiar finding in our study was that HCWs practicing in a public health facility were more likely to express a higher level of PB and WB. This could be explained that limited work resources may increase job demands and the negative psychological/physiological costs associated with them, such as burnout (56) which is the case of public health facilities. However, such a finding was anticipated since public hospitals were the first facilities to be mobilized by the ministry of public health for the fight against COVID-19.

Age and altruism emerged in our study as protective factors where burnout levels in HCWs tend to decrease with increasing age and with altruism. This could be due to the more "perfectionistic" and empathic approach in younger HCWs (57). As for altruism and its negative association with burnout, previous theories in psychology considered that altruistic behavior has dominance over negative emotions and anxiety. This will increase engagement in altruistic activities in challenging situations(58).

Extensive working hours and insufficient sleeping hours were also found associated with high level of burnout in all its aspects. This could be understood since the workload imposed by the pandemic leads to long working hours and short sleeping hours which are common factors associated with burnout. A study assessing the relationship between sleeping hours and burnout showed that the odds ratio of workrelated burnout doubled when hours exceeded 60 hours, tripled when hours exceeded 74 hours, and quadrupled when hours exceeded 84 hours (59).

Lastly, low income was found as a factor increasing burnout in all its aspects. As known, socioeconomic status can refer to a person's quality of life as well as the opportunities and privileges they have in society. Several studies reported that financial strain was associated with high

burnout (60) and socioeconomic status was found as a constant and reliable predictor of a wide range of life outcomes, including physical and mental health. However, some issues should be highlighted in the case of Lebanese HCWs who faced an unprecedented economic crisis. The latter has affected severely HCW's income as a result of the depletion of the Lebanese currency. This has increased HCWs' feelings of insecurity towards their work which could occupy their mind and make them lose focus. In addition, they can feel more hopeless and dissatisfied with their work and their patients. It is worth mentioning that having a low income in our study referred to the current self-perceived economic situation. The finding that HCWs who experienced a deteriorated economic situation (low income) during the study time also reported increasing burnout levels indicates that subjective economic difficulties might have an impact on burnout.

Although the relationship between burnout and the COVID-19 pandemic has been unveiled in this study, several potential factors associated with burnout among Lebanese HCWs were not investigated in our study. Hence, further research about other risk factors that could be incremental, in particular factors related to economic factors, and how to alleviate burnout symptoms among HCWs fighting against COVID-19 is still needed

\section{Limitations:}


There are several limitations to this study we should address. First, the cross-sectional design of the study limits our ability to infer causal relationships. In addition, the collected data were also based on self-reported information which makes it prone to social desirability and might lead to underestimating some associations. Secondly, selection bias is possible due to the sampling technique used for data collection which limits the generalizability of the findings. Thirdly, our study data were collected using an online questionnaire. Although a substantial number of HCWs from different regions across Lebanon during the current outbreak of COVID-19 were able to participate and the good quality of data collected by online surveys, some drawbacks related to the online nature of the survey should be acknowledged. HCWs with who were busy with higher levels of workload, and possibly with a higher risk of being infected at work, may not have the time to fill out the survey. Additionally, HCWs with limited internet access may not have taken part in this study. Fourthly, we were unable to assess the pandemic's impact on burnout due to a lack of data on pre-COVID burnout among Lebanese HCWs using the same assessment tool. Lastly, further studies following up on the burnout of Lebanese physicians would be recommended in the future to confirm our results, especially since several waves of COVID-19 have been recorded since December 2020.

\section{Implications for Clinical Practice and Research}

The present study has relevant practical implications, in terms of burnout prevention, for hospitals and HCWs. Occupational risks were found in several studies to be associated with a higher workload. This could be explained that the threatening situations requiring additional efforts and tasks to be managed (61). Hence to prevent burnout among HCWs in the hospital setting, we should consider the perceived threat of being infected by COVID-19 as an additional work demand for HCWs requiring an investment of further energies at physical and psychological levels. Hence, hospitals were encouraged to adjust the balance between job demands and available resources. This includes ensuring the availability of adequate protective equipment and effective safety-related policies, exchange of reliable information about COVID-19 risk across HCWs in addition to monitoring of implemented precautionary measures. On the one hand, The latter will allow controlling the work environment and achieving effectively safety at the workplace, thus allowing HCWs to feel safe and able to cope efficiently with the perceived threat of COVID-19. This will reduce pandemic-related burnout among them. On other hand, the alarming level of burnout unveiled among Lebanese HCWs represented only the tip of the iceberg where migration of a huge number of HCWs especially physicians and nurses was noticed in the previous months. Projections

show that the health workforce shortage is expected to increase over the coming months. The latter could threaten the patient's quality of care and the overall healthcare system. Such a high level of burnout underlines the urgent need that government and health facilities address this comorbidity through enacting proactive policies, providing critical leadership and funding for burnout prevention programs. A collaborative effort between national and institutional leadership will improve burnout management during this pandemic and better prepare us for the future. Since the health care workforce is an indispensable part of the economic growth and resilience of a nation, policymakers should be pragmatic 
in supporting funding for burnout prevention programs. Lastly, the long-term effects of the current pandemic need to be assessed later.

\section{Conclusions}

The alarming level of burnout detected among Lebanese HCWs in all its three aspects( personal, occupational, and client-related) calls for urgent action. Health authorities should be proactive and address the factors associated with burnout unveiled in our study. Enacting and implementing preventive policies and effective evidence-based interventions are highly required to cultivate wellness among HCWs to reduce their burnout. This could slow down the ongoing attrition of HCWs, prevent possible detrimental consequences for HCW's well-being and ensure all patients receive quality care from motivated and hopeful healthcare providers. Forthcoming studies that investigate additional situational and individual factors that may affect burnout are recommended.

\section{Declarations}

\section{Author Contributions:}

D.Y. was involved with study conception and design, data collection and analysis, drafted and revised the manuscript.

\section{L.A.A. was involved in revising the article.}

H.H. and E.A. were involved in the study conceptualization and design and revising the article.

J.Y. was involved in the study conceptualization and the revision of the manuscript.

All authors have read and agreed to the published version of the manuscript.

\section{Funding:}

This research received no external funding.

\section{Informed Consent Statement:}

Informed consent for participating in the study was obtained digitally through Google Forms from all subjects, and all methods were carried out as per the relevant guidelines and national regulations for the Non-clinical studies. Specifically, at the beginning of the questionnaire, participants were asked whether they agree to participate in the research to be included in the study. Participants were also informed that their participation was voluntary and that they had the right to leave at any time without providing any explanation. No incentives were provided to the study participants. 


\section{Data Availability Statement:}

The datasets generated during the current study are not publicly available but are available from the corresponding author on reasonable request.

\section{Conflicts of Interest}

The authors declare that they have no competing interests and they have no known competing financial interests or personal relationships that could have appeared to influence the work reported in this paper.

\section{Consent for publication}

Not applicable.

\section{Authors information}

Dalal Youssef, MSc, MPH, RSM, Ph.D., Institut de santé publique, d'épidémiologie et de développement (ISPED), Bordeaux University, France \& Clinical trial Program, Ministry of Public Health, Lebanon.

Edmond Abboud, MD, Ph.D., Ministry of Public Health, Beirut, Lebanon

Linda Abou Abbas, MPH, Ph.D., Neuroscience Research Center, Lebanese University, Faculty of Medical Sciences, Beirut, Lebanon

Janet Youssef, MD, OBGYN, Al Zahraa Hospital University Medical Center, Beirut, Lebanon (ZHUMC), Lebanon

Hamad Hassan, PharmD, Ph.D., Ministry of Public Health, Beirut, Lebanon

\section{Abbreviations}

COVID-19: Coronavirus Disease 2019

CBI: Copenhagen Burnout Inventory

TP: Threat perception scale

HCW: Health care worker

PB: personal burnout

WB: Work-related burnout 
CB: client-related burnout

MOPH: Ministry of Public Health

aOR: Adjusted odds ratio

EMR: Eastern Mediterranean Region

\section{References}

1. Dubey S, Biswas P, Ghosh R, Chatterjee S, Dubey MJ, Chatterjee S, et al. Psychosocial impact of COVID-19. Diabetes \& metabolic syndrome. 2020;14(5):779-88.

2. Susskind D, Vines DJORoEP. The economics of the COVID-19 pandemic: an assessment. 2020;36(Supplement_1):S1-S13.

3. Xiong J, Lipsitz O, Nasri F, Lui LM, Gill H, Phan L, et al. Impact of COVID-19 pandemic on mental health in the general population: A systematic review. 2020.

4. Spoorthy MS, Pratapa SK, Mahant S. Mental health problems faced by healthcare workers due to the COVID-19 pandemic-A review. Asian J Psychiatr. 2020;51:102119-.

5. Ghaleb Y, Lami F, Al Nsour M, Rashak HA, Samy S, Khader YS, et al. Mental health impacts of COVID-19 on healthcare workers in the Eastern Mediterranean Region: a multi-country study. Journal of Public Health. 2021;43(Supplement_3):iii34-iii42.

6. Robertson E, Hershenfield K, Grace SL, Stewart DEJTCJoP. The psychosocial effects of being quarantined following exposure to SARS: a qualitative study of Toronto health care workers. 2004;49(6):403-7.

7. Gupta S, Sahoo S. Pandemic and mental health of the front-line healthcare workers: a review and implications in the Indian context amidst COVID-19. Gen Psychiatr. 2020;33(5):e100284-e.

8. Cyr S, Marcil M-J, Marin M-F, Tardif J-C, Guay S, Guertin M-C, et al. Factors Associated With Burnout, Post-traumatic Stress and Anxio-Depressive Symptoms in Healthcare Workers 3 Months Into the COVID-19 Pandemic: An Observational Study. Front Psychiatry. 2021;12:668278-.

9. Maslach C, Leiter MP. Understanding the burnout experience: recent research and its implications for psychiatry. World Psychiatry. 2016;15(2):103-11.

10. Bunjak A, Černe M, Nagy N, Bruch H. Job demands and burnout: The multilevel boundary conditions of collective trust and competitive pressure. Human Relations. 2021:00187267211059826.

11. Shreffler J, Petrey J, Huecker M. The Impact of COVID-19 on Healthcare Worker Wellness: A Scoping Review. West J Emerg Med. 2020;21(5):1059-66. 
12. Søvold LE, Naslund JA, Kousoulis AA, Saxena S, Qoronfleh MW, Grobler C, et al. Prioritizing the Mental Health and Well-Being of Healthcare Workers: An Urgent Global Public Health Priority. 2021;9.

13. Leo CG, Sabina S, Tumolo MR, Bodini A, Ponzini G, Sabato E, et al. Burnout Among Healthcare Workers in the COVID 19 Era: A Review of the Existing Literature. Front Public Health. 2021;9:750529-.

14. Salvagioni DAJ, Melanda FN, Mesas AE, González AD, Gabani FL, Andrade SMd. Physical, psychological and occupational consequences of job burnout: A systematic review of prospective studies. PLoS One. 2017;12(10):e0185781-e.

15. Hall LH, Johnson J, Watt I, Tsipa A, O'Connor DB. Healthcare Staff Wellbeing, Burnout, and Patient Safety: A Systematic Review. PLoS One. 2016;11(7):e0159015-e.

16. Motluk A. Do doctors experiencing burnout make more errors? CMAJ. 2018;190(40):E1216-E7.

17. Bayani DBS, Tan SG. Health Systems Impact of COVID-19 in the Philippines. 2021.

18. Bank W. Lebanon Economic Monitor, Fall 2020: The Deliberate Depression. World Bank; 2020.

19. Farran N. Mental health in Lebanon: Tomorrow's silent epidemic. Mental Health \& Prevention. 2021;24:200218.

20. Bank W. Lebanon Economic Monitor, Spring 2021: Lebanon Sinking (to the Top 3). World Bank; 2021.

21. Ministry of Public Health Esu. COVID-19 surveillance in Lebanon-daily report. 2020(30 December 2020).

22. Sabbah I, Sabbah H, Sabbah S, Akoum H, Droubi N. Burnout among Lebanese nurses:

Psychometric properties of the Maslach Burnout Inventory-Human Services Survey (MBI-HSS). Health. 2012;4:644-65249101.

23. Talih F, Warakian R, Ajaltouni J, Shehab AA, Tamim H. Correlates of Depression and Burnout Among Residents in a Lebanese Academic Medical Center: a Cross-Sectional Study. Academic psychiatry : the journal of the American Association of Directors of Psychiatric Residency Training and the Association for Academic Psychiatry. 2016;40(1):38-45.

24. Clinton M, Shehadeh MSNRNR. Rasch Analysis of Lebanese Nurses' Responses to the Copenhagen Burnout Inventory Alternative to the Maslach Burnout Inventory. SAGE Open Nursing. $2021 ; 7: 23779608211020919$.

25. Bizri M, Kassir G, Tamim H, Kobeissy F, Hayek SE. Psychological distress experienced by physicians and nurses at a tertiary care center in Lebanon during the COVID-19 outbreak. Journal of health psychology. 2021:1359105321991630. 
26. Chong MY, Wang WC, Hsieh WC, Lee CY, Chiu NM, Yeh WC, et al. Psychological impact of severe acute respiratory syndrome on health workers in a tertiary hospital. The British journal of psychiatry : the journal of mental science. 2004;185:127-33.

27. Msheik El Khoury F, Talih F, Khatib MFE, Abi Younes N, Siddik M, Siddik-Sayyid S. Factors Associated with Mental Health Outcomes: Results from a Tertiary Referral Hospital in Lebanon during the COVID-19 Pandemic. Libyan Journal of Medicine. 2021;16(1):1901438.

28. Dalal Y, Linda A-A, Janet Y. Research Square. 2022.

29. Huo L, Zhou Y, Li S, Ning Y, Zeng L, Liu Z, et al. Burnout and Its Relationship With Depressive Symptoms in Medical Staff During the COVID-19 Epidemic in China. Frontiers in psychology. 2021;12:616369.

30. Khasne RW, Dhakulkar BS, Mahajan HC, Kulkarni AP. Burnout among Healthcare Workers during COVID-19 Pandemic in India: Results of a Questionnaire-based Survey. Indian J Crit Care Med. 2020;24(8):664-71.

31. Matsuo T, Kobayashi D, Taki F, Sakamoto F, Uehara Y, Mori N, et al. Prevalence of Health Care Worker Burnout During the Coronavirus Disease 2019 (COVID-19) Pandemic in Japan. JAMA Network Open. 2020;3(8):e2017271-e.

32. Alsulimani LK, Farhat AM, Borah RA, AlKhalifah JA, Alyaseen SM, Alghamdi SM, et al. Health care worker burnout during the COVID-19 pandemic: A cross-sectional survey study in Saudi Arabia. Saudi medical journal. 2021;42(3):306-14.

33. AlHumaid J, Ali S, Farooq IJPTT, Research, Practice, Policy. The psychological effects of the COVID-19 pandemic and coping with them in Saudi Arabia. 2020;12(5):505.

34. Barello S, Palamenghi L, Graffigna GJPr. Burnout and somatic symptoms among frontline healthcare professionals at the peak of the Italian COVID-19 pandemic. 2020;290:113129.

35. Lasalvia A, Bonetto C, Porru S, Carta A, Tardivo S, Bovo C, et al. Psychological impact of COVID-19 pandemic on healthcare workers in a highly burdened area of north-east Italy. 2021;30.

36. Di Trani M, Mariani R, Ferri R, De Berardinis D, Frigo MG. From Resilience to Burnout in Healthcare Workers During the COVID-19 Emergency: The Role of the Ability to Tolerate Uncertainty. 2021;12.

37. Duarte I, Teixeira A, Castro L, Marina S, Ribeiro C, Jácome C, et al. Burnout among Portuguese healthcare workers during the COVID-19 pandemic. 2020;20(1):1-10.

38. Elhadi M, Msherghi A, Elgzairi M, Alhashimi A, Bouhuwaish A, Biala M, et al. Burnout syndrome among hospital healthcare workers during the COVID-19 pandemic and civil war: a cross-sectional study. 2020;11:1426. 
39. Rotenstein LS, Torre M, Ramos MA, Rosales RC, Guille C, Sen S, et al. Prevalence of burnout among physicians: a systematic review. 2018;320(11):1131-50.

40. Žutautienè R, Radišauskas R, Kaliniene G, Ustinaviciene RJljoer, health $p$. The prevalence of burnout and its associations with psychosocial work environment among Kaunas region (Lithuania) hospitals' physicians. 2020;17(10):3739.

41. Shanafelt TD. Enhancing Meaning in Work: A Prescription for Preventing Physician Burnout and Promoting Patient-Centered Care. JAMA. 2009;302(12):1338-40.

42. Houkes I, Winants Y, Twellaar M, Verdonk P. Development of burnout over time and the causal order of the three dimensions of burnout among male and female GPs. A three-wave panel study. BMC Public Health. 2011;11(1):240.

43. Gramaglia C, Marangon D, Azzolina D, Guerriero C, Lorenzini L, Probo M, et al. The Mental Health Impact of 2019-nCOVID on Healthcare Workers From North-Eastern Piedmont, Italy. Focus on Burnout. 2021;9:483.

44. Prasad K, McLoughlin C, Stillman M, Poplau S, Goelz E, Taylor S, et al. Prevalence and correlates of stress and burnout among U.S. healthcare workers during the COVID-19 pandemic: A national crosssectional survey study. EClinicalMedicine. 2021;35:100879.

45. Shanafelt TD, Boone S, Tan L, Dyrbye LN, Sotile W, Satele D, et al. Burnout and satisfaction with work-life balance among US physicians relative to the general US population. 2012;172(18):1377-85.

46. Hodzi MB, Annor F, Darkwah E. An exploration of work-related experiences of domestic workers in Accra, Ghana. Journal of Workplace Behavioral Health. 2021;36(4):309-34.

47. Gauche C, de Beer LT, Brink LJSJoHRM. Managing employee well-being: A qualitative study exploring job and personal resources of at-risk employees. 2017;15:13.

48. Blanch A, Aluja AJHr. Social support (family and supervisor), work-family conflict, and burnout: Sex differences. 2012;65(7):811-33.

49. Parreñas RSJRYS. Women, migration and domestic work. 2001;15(28.2003).

50. Maher J. Nurse mothers valuing care at home and at work: Beyond notions of care scarcity? Journal of Sociology. 2012;50(4):531-44.

51. Lai J, Ma S, Wang Y, Cai Z, Hu J, Wei N, et al. Factors Associated With Mental Health Outcomes Among Health Care Workers Exposed to Coronavirus Disease 2019. JAMA Network Open. 2020;3(3):e203976-e. 
52. Nahrgang JD, Morgeson FP, Hofmann DAJJoap. Safety at work: a meta-analytic investigation of the link between job demands, job resources, burnout, engagement, and safety outcomes. 2011;96(1):71.

53. Day AL, Sibley A, Scott N, Tallon JM, Ackroyd-Stolarz SJCJoASRCdSdlA. Workplace risks and stressors as predictors of burnout: The moderating impact of job control and team efficacy. 2009;26(1):722.

54. Nielsen MB, Mearns K, Matthiesen SB, Eid JJSJoP. Using the Job Demands-Resources model to investigate risk perception, safety climate and job satisfaction in safety critical organizations. 2011;52(5):465-75.

55. Xiao X, Zhu X, Fu S, Hu Y, Li X, Xiao JJJoad. Psychological impact of healthcare workers in China during COVID-19 pneumonia epidemic: A multi-center cross-sectional survey investigation. 2020;274:40510.

56. Xanthopoulou D, Bakker AB, Dollard MF, Demerouti E, Schaufeli WB, Taris TW, et al. When do job demands particularly predict burnout? The moderating role of job resources. 2007.

57. Wiederhold BK, Cipresso P, Pizzioli D, Wiederhold M, Riva GJOM. Intervention for physician burnout: a systematic review. 2018;13(1):253-63.

58. Post SG. Altruism, happiness, and health: it's good to be good. International Journal of Behavioral Medicine. 2005;12(2):66-77.

59. Lin RT, Lin YT, Hsia YF, Kuo CC. Long working hours and burnout in health care workers: Non-linear dose-response relationship and the effect mediated by sleeping hours-A cross-sectional study. Journal of occupational health. 2021;63(1):e12228.

60. Soares JJ, Grossi G, Sundin O. Burnout among women: associations with demographic/socioeconomic, work, life-style and health factors. Archives of women's mental health. 2007;10(2):61-71.

61. Leiter MPJA, Stress, Coping. Perception of risk: An organizational model of occupational risk, burnout, and physical symptoms. 2005;18(2):131-44.

\section{Figures}




\section{HCWs' perception of the COVID-19 pandemic threat}



Figure 1

HCWs' perception of the COVID-19 threat

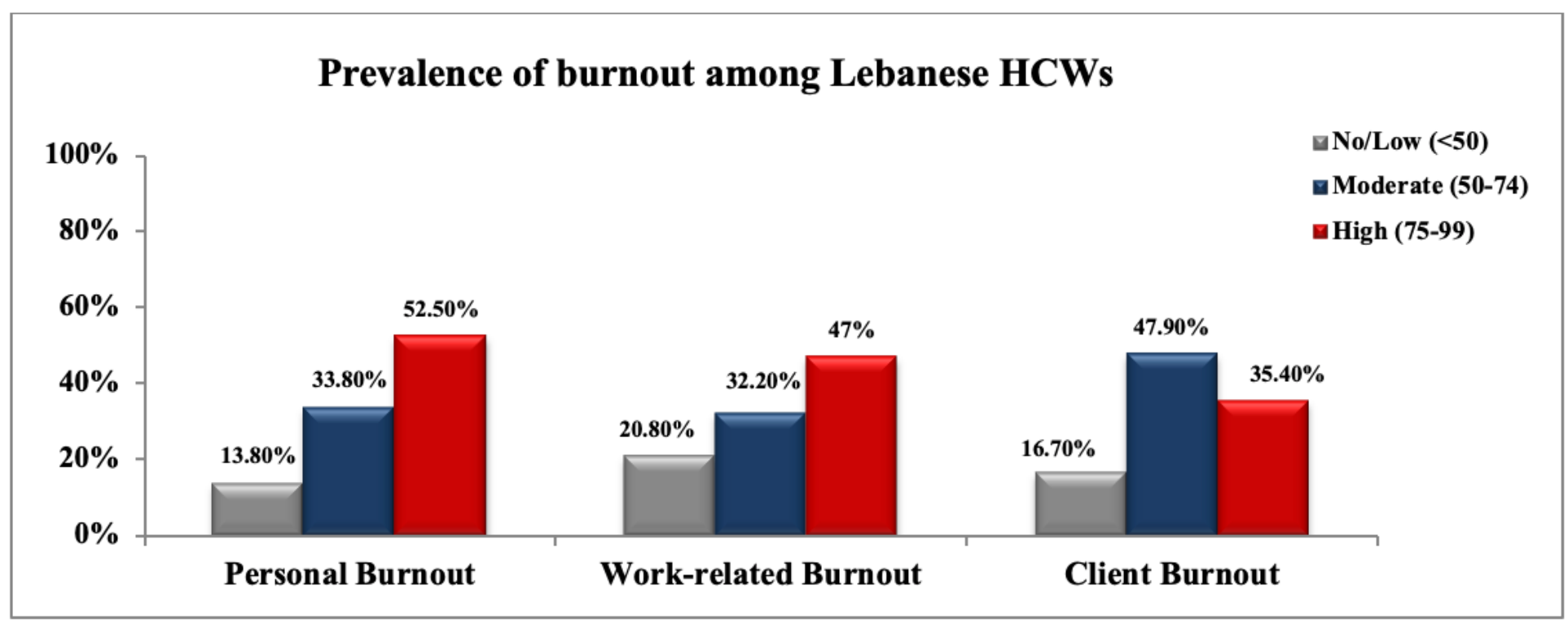

Figure 2

Prevalence of the three aspects of burnout among Lebanese HCWs 